\title{
In Vivo Testing of a Novel Adjustable Glaucoma Drainage Device
}

\author{
Adan Villamarin, ${ }^{1}$ Nikos Stergiopulos,${ }^{1}$ Stéphane Bigler, ${ }^{1}$ André Mermoud, ${ }^{2}$ Alexandre Moulin, ${ }^{3}$ \\ and Sylvain Roy ${ }^{1,2}$
}

${ }^{1}$ Swiss Federal Institute of Technology, Lausanne, Switzerland

${ }^{2}$ Glaucoma Center Montchoisi Clinic, Lausanne, Switzerland

3 Jules Gonin Eyes Hospital, Lausanne, Switzerland

Correspondence: Adan Villamarin, Laboratory of Hemodynamics and Cardiovascular Technology, LHTC, EPFL, STI - IBI - LHTC, Station 17, 1015 Lausanne, Switzerland; adan.villamarin@epfl.ch.

Submitted: April 10, 2014 Accepted: September 25, 2014

Citation: Villamarin A, Stergiopulos N, Bigler S, Mermoud A, Moulin A, Roy S In vivo testing of a novel adjustable glaucoma drainage device. Invest Ophthalmol Vis Sci. 2014;55:75207524. DOI:10.1167/iovs.14-14563
Purpose. We report on the in vivo testing of a novel noninvasively adjustable glaucoma drainage device (AGDD), which features an adjustable outflow resistance, and assess the safety and efficiency of this implant.

Methods. Under general anesthesia, the AGDD was implanted on seven white New Zealand rabbits for a duration of 4 months under a scleral flap in a way analogous to the Ex-PRESS device and set in an operationally closed position. The IOP was measured on a regular basis on the operated and control eyes using a rebound tonometer. Once a month the AGDD was adjusted noninvasively from its fully closed to its fully open position and the resulting pressure drop was measured. The contralateral eye was not operated and served as control. After euthanization, the eyes were collected for histology evaluation.

Results. The mean preoperative IOP was $11.1 \pm 2.4 \mathrm{~mm} \mathrm{Hg}$. The IOP was significantly lower for the operated eye $(6.8 \pm 2 \mathrm{~mm} \mathrm{Hg})$ compared to the nonoperated eye $(13.1 \pm 1.6 \mathrm{~mm} \mathrm{Hg})$ during the first 8 days after surgery. When opening the AGDD from its fully closed to fully open position, the IOP dropped significantly from $11.2 \pm 2.9$ to $4.8 \pm 0.9 \mathrm{~mm} \mathrm{Hg}(P<0.05)$.

Concuusions. Implanting the AGDD is a safe and uncomplicated surgical procedure. The fluidic resistance was noninvasively adjustable during the postoperative period with the AGDD between its fully closed and fully open positions.

Keywords: drainage device, aqueous shunt, filtering surgery, glaucoma drainage device, aqueous humor
$\mathrm{G}$ laucoma is a leading cause of blindness affecting several million patients worldwide. ${ }^{1,2}$ Decreasing the IOP is one of the most important requirements to control the progression of glaucoma efficiently. ${ }^{3}$ The number of valuable options in glaucoma filtering surgery has increased over the last decade. ${ }^{4,5}$ Trabeculectomy and the use of implantable drainage devices or stents are parts of the armamentarium available nowadays in modern glaucoma filtering procedures. Glaucoma drainage implants become increasingly popular because they may offer better IOP control over time, reducing the number of postoperative complications. ${ }^{6,7}$ Still, predicting postoperative IOP and maintaining it at optimal levels remains a key problem. For instance, the risk of early postoperative complications, such as hypotony, always is present. Hypotony could entrain complications requiring further surgeries. ${ }^{8,9}$ In that respect, the possibility to control the fluidic resistance of an implanted drainage device during the postoperative follow-up would have been very helpful in maintaining IOP always at optimal levels, thereby potentially avoiding a number of complications related to poor pressure control.

Recently, a new experimental noninvasively adjustable glaucoma drainage device (AGDD) has been developed. ${ }^{10}$ In vitro results demonstrated that the fluidic resistance of the device can be adjusted to consequently regulate the IOP. To assess the safety and the efficiency of the novel device, we report the first in vivo testing of this AGDD.

\section{Materials AND Methods}

The principle of the AGDD system was first described by Stergiopulos, ${ }^{11}$ further developed and patented by Bigler ${ }^{12}$ and Stergiopulos, ${ }^{11}$ and described in details by Villamarin et al. ${ }^{10}$ Briefly, the system comprises the AGDD implant and the external measurement/adjustment device, hereafter called the control unit (CU; Fig. 1). The AGDD contains a simple mechanism, which allows for a selective compression of an incorporated drainage tube, thereby adjusting its fluidic resistance. This is achieved using the combination of an eccentrically placed magnetic disk (that is placed on ball bearings facilitating its rotation) and a ring compressing the silicone tube; the angle of rotation, thus, determines the amount of compression of the drainage tube, and, therefore, its fluidic resistance. The magnetic disk is rotated using the $\mathrm{CU}$, which contains a magnet at one end and a compass on the other end. When placed on top of the implanted AGDD, the compass indicates the actual rotational position of the magnetic disk. The magnet is used to rotate the magnetic disk, by appropriately dragging the magnet along the periphery of the implant in the clockwise or counterclockwise direction. The reading (compass) and the adjustment (magnet) of the rotational position of the implant are done noninvasively and without even touching the eye (Fig. 1). 


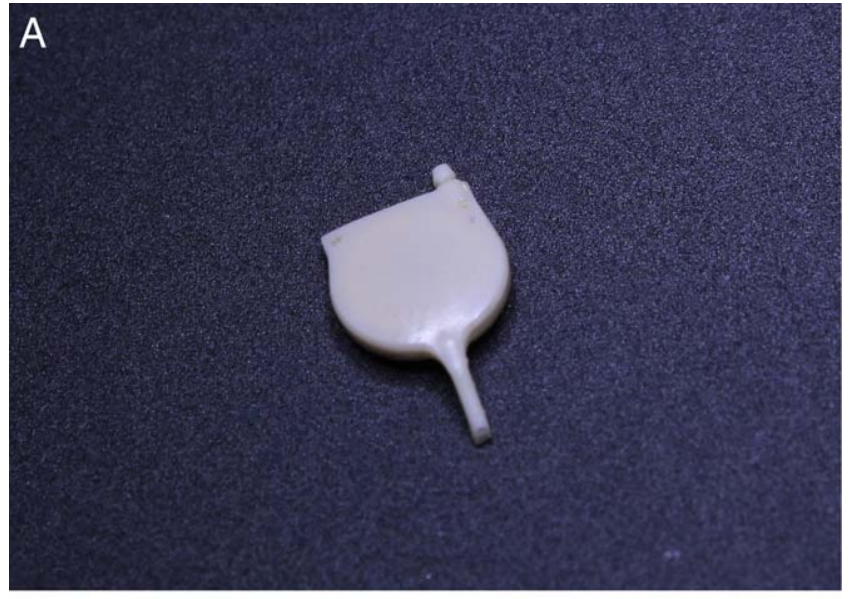

B

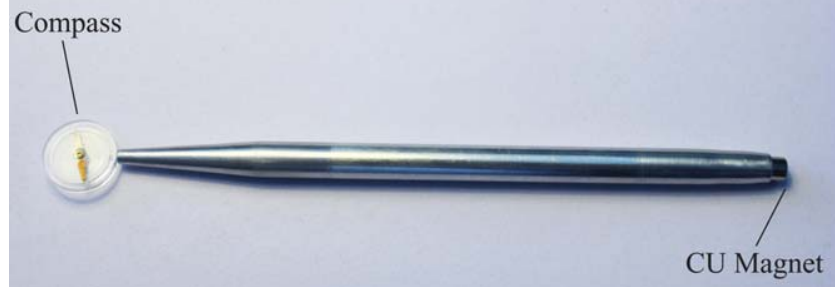

CU Magnet

Figure 1. Picture of the AGDD (A) and the CU (B) used for the measurement and the adjustment of the outflow resistance.

\section{In Vivo Experiment}

The study was done on seven white New Zealand rabbits for duration of 4 months. General anesthesia was performed using a mixture of $3 \mathrm{mg} / \mathrm{kg}$ (body mass) xylazine and $35 \mathrm{mg} / \mathrm{kg}$ ketamine intramuscular injection. Under general anesthesia, the AGDD was implanted in a way analogous to the Ex-PRESS device. ${ }^{13}$ In short, a scleral flap of $7 \times 7 \mathrm{~mm}$ (width $\times$ long) was dissected and the nozzle was inserted through a paracentesis made by a 25-gauge needle in the anterior chamber between the cornea and the anterior surface of the iris taking care not to touch either of these structures (Fig. 2). The AGDD was implanted in a functionally closed position and left in that position during the initial postoperative period (1 month). The end portion of the tube was placed freely under the conjunctiva and aqueous humor was drained in the subconjunctival space.

During the first postoperative week, IOP was measured daily on the operated and control eyes using a rebound tonometer (iCARE Tonovet; iCare, Vanda, Finland). In the following weeks, such measurement was performed once a week. Results were reported and compared for statistics. Before each measurement, rabbits were placed in a bunny snuggle (Colonial Medical Supply, Nashua, NH, USA) and relaxed for 5 minutes. All measurements were performed at the end of the morning (10 AM to 12 noon).

Once a month, the AGDD was adjusted noninvasively with the CU from its fully closed position to its fully open position and the resulting pressure drop was measured. Hypotony was defined as an IOP equal or lower than $6 \mathrm{~mm} \mathrm{Hg}$ (IOP $\leq 6 \mathrm{~mm}$

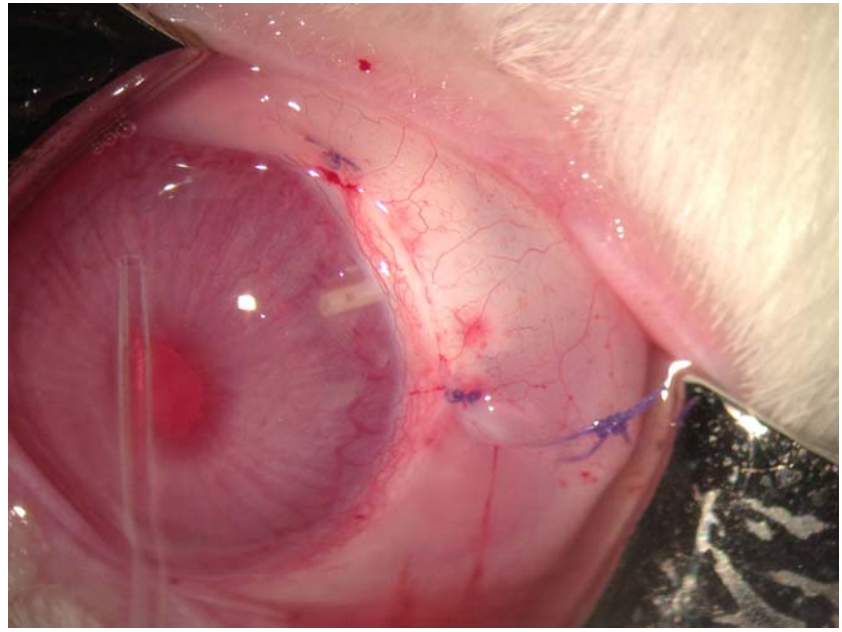

FIGURE 2. Picture of a rabbit eye where the AGDD was placed under a scleral flap with its nozzle inserted in the anterior chamber.

$\mathrm{Hg}$ ). The contralateral eye was not operated and served as control. At the end of the study, the animals were euthanized and the eyes were enucleated to perform histology to assess the biocompatibility of the AGDD.

All experiments conformed to the ARVO Statement for the Use of Animals in Ophthalmic and Vision Research, and were reviewed and approved by the institutional committee for animal studies in Lausanne, Switzerland.

\section{Histology}

The enucleated eyes were formalin-fixed, macroscopically processed, and dehydrated through graded alcohol followed by paraffin inclusion. Sections $(5 \mu \mathrm{m})$ were cut and stained with hematoxylin-eosin and Prussian blue. The local inflammatory response was assessed using the following parameters: (1) density of lymphocytic and plasmacytic infiltrate around the AGDD device $(\mathrm{X}=$ number of cells $/ 5$ high power field [HPF], $\times 400,1.8 \mathrm{~mm}^{2}$ ), (2) density of eosinophilic infiltrate (number of cells/5 HPF), (3) intensity of granulomatous inflammation, and (4) fibrosis and scarring. A cellular density $<10$ cells $/ 5$ HPF was graded as minimal, $10>\mathrm{X}<50$ cells $/ 5$ $\mathrm{HPF}$ as mild, and $\mathrm{X}>50$ cells $/ 5 \mathrm{HPF}$ as marked inflammation. The intensity of granulomatous reaction was graded as minimal with only few macrophages and giant cells, moderate with more granuloma, and marked with sheets of macrophages and giant cells. The fibrosis and scarring was graded as minimal if the scarring maximal thickness was inferior to $25 \%$ of the scleral thickness, mild $<50 \%$, and moderate $>50 \%$.

\section{Statistics}

The results were expressed as the mean and SD. The paired Student's $t$-test was used to assess significant differences and effects of the AGDD on the results. $P<0.05$ was considered statistically significant.

\section{Results}

The mean preoperative IOP was $11.1 \pm 2.4 \mathrm{~mm} \mathrm{Hg}$. The evolution of IOP during the entire postoperative period for the animals is shown in Figure 3. For the seven rabbits, hypotony was present in the operated eyes during the first 3 days after implantation (mean IOP was $6.0 \pm 1.4 \mathrm{~mm} \mathrm{Hg}$ ) and the difference with the control (nonoperated) eyes was significant 


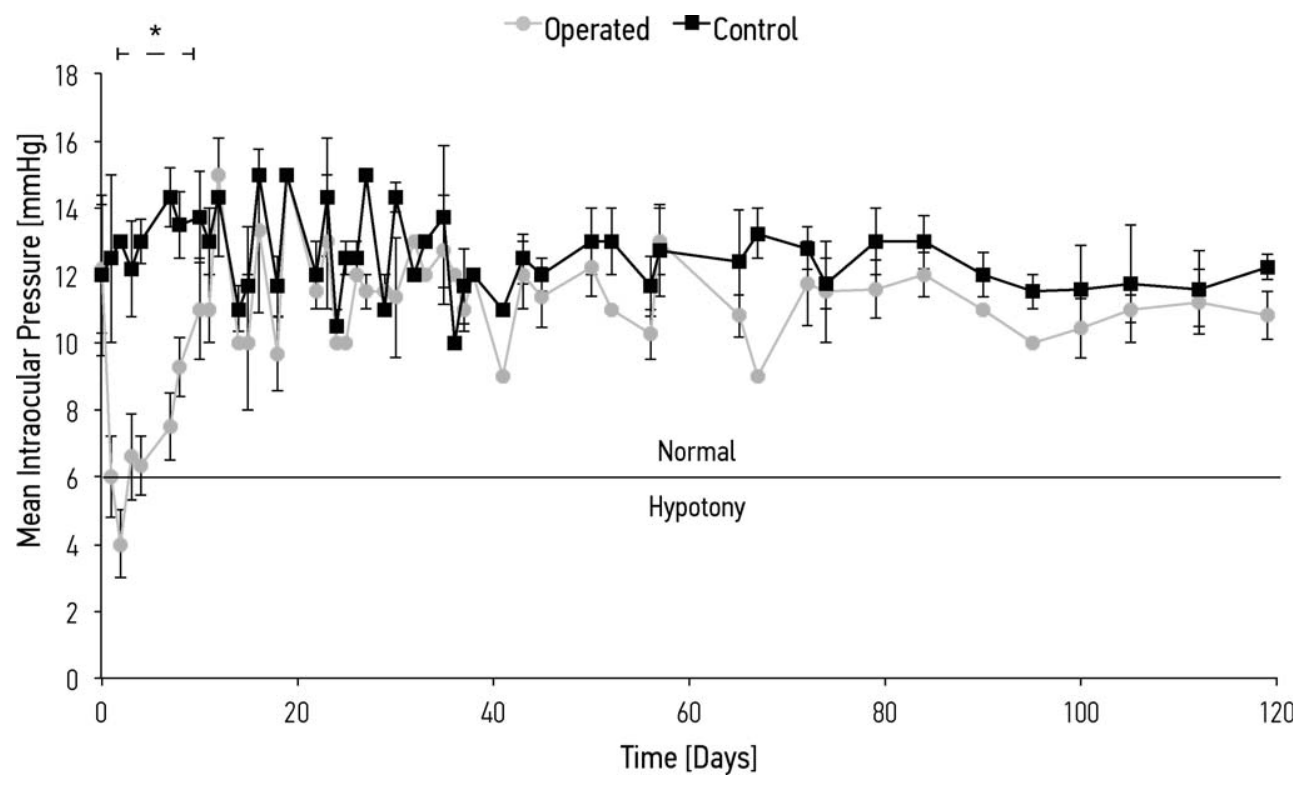

FigurE 3. Plot of the mean IOP over time. Measurements were done every week with an iCARE TonoVet. Postoperatively the difference between the operated and control eyes was significant from days 1 to $8\left(^{*}\right)$. Transient hypotony was present in the operated eyes during the first 3 days after implantation.

$(P<0.05)$. Eight days after surgery, hypotony was no longer present (mean IOP, $11.3 \pm 0.8 \mathrm{~mm} \mathrm{Hg}$ ), and the difference in IOP between control and operated eyes was no longer significant $(P=0.12$, Fig. 3). In all rabbits, the IOP dropped significantly from $11.2 \pm 2.9$ to $4.8 \pm 0.9 \mathrm{~mm} \mathrm{Hg}(P<0.05)$ when the AGDD was opened from its fully closed (maximum outflow resistance) to fully opened (minimum outflow resistance) position (Fig. 4). The range of IOP means from the functional closed position of the AGDD to the functional open position corresponded to: closed position, minimum $=7$ $\mathrm{mm} \mathrm{Hg}$, maximum $=17 \mathrm{~mm} \mathrm{Hg}$; and open position, minimum $=4 \mathrm{~mm} \mathrm{Hg}$, maximum $7 \mathrm{~mm} \mathrm{Hg}$. The pressure drop was significant for all rabbits $(P<0.05$, Fig. 5).

Microscopic examination of tissues surrounding the AGDD showed a cavity covered by a layer of flattened macrophages surrounded by partially reorganized dense connective tissue. In some areas, aggregates of lymphocytes as well as scarce eosinophils could be identified. The chronic inflammatory infiltrate was minimal to mild in most cases (Fig. 6). More precisely, the lymphocytic and plasmocytic inflammatory infiltrate was mild in all cases. The eosinophilic infiltrate was minimal in two cases and mild in five cases. A granulomatous foreign body type reaction was observed around the suture material. The intensity of the granulomatous reaction was minimal to mild in five cases and mild to moderate in two cases. The scarring and fibrous reaction was mild to moderate in all cases. Finally, there was no intraocular inflammation, no alteration of the retinal architecture, and no atrophy of the ganglion cells layers present in any case. The optic nerves were unremarkable in all cases.

\section{Discussion}

The advantage of the novel glaucoma drainage device resides in the possibility to adjust, noninvasively, its fluidic resistance to the aqueous humor drainage. In that prospect, the goal of this study was to demonstrate the safety, efficacy, ease of use, and biocompatibility of the AGDD in an animal model.

Although rabbits are standard animal models commonly used in ophthalmology research, ${ }^{14}$ the size of the rabbit eye is significantly smaller than in humans. However, the anatomical structures are comparable, providing a useful surgical model for ophthalmic investigations. ${ }^{15}$ The rabbits used in this study did not suffer from glaucoma. It is rather difficult to induce glaucoma on rabbit eyes and to maintain an elevated IOP over time. Several techniques, such as injection of microbeads into the anterior chamber ${ }^{16}$ or occlusion of episcleral vessels, ${ }^{17,18}$ can be effective, but do not work for long-term follow-ups. In addition, these techniques were tried on rodents, such as mice or rats, that have too small eyes for the implantation of the AGDD. Therefore, the capability in reducing elevated IOP to normal range (e.g. in patients suffering from glaucoma) could not be tested in this study. However, the ability of the AGDD to reduce pressure levels (from normal to low pressures) has been demonstrated clearly. Because the implant operates on altering the fluidic resistance, one could imagine that in presence of glaucomatous eyes, the IOP could be reduced to pressures down to physiological range. Results showed that the implant has efficiently decreased the IOP once it was in its functionally open position. These results demonstrated that over time the AGDD can be adjusted noninvasively and consequently the resistance to outflow can be adjusted accordingly. When the implant was readjusted from the fully open to its fully closed position, the following day, the IOP resumed its initial level (Fig. 4). These observations are very encouraging, demonstrating the ability of the implant to change the outflow facility and to help control the IOP at the level of the tube. However, the relatively narrow IOP range with this normotensive rabbit model makes the reading of the IOP drop more difficult when the AGDD is set on partially opened positions. Further clinical studies with glaucomatous eyes having greater IOP range should confirm that partially opened positions would lead to a controlled IOP drop.

The surgical method to implant the AGDD is analogous to the Ex-PRESS tube, which is comparable to the trabeculectomy technique. ${ }^{19}$ Nevertheless, some minor changes were necessary to correctly implant the AGDD. For instance, the size of the scleral flap was substantially larger $(7 \times 7 \mathrm{~mm})$ to correctly fit the size of the AGDD. 


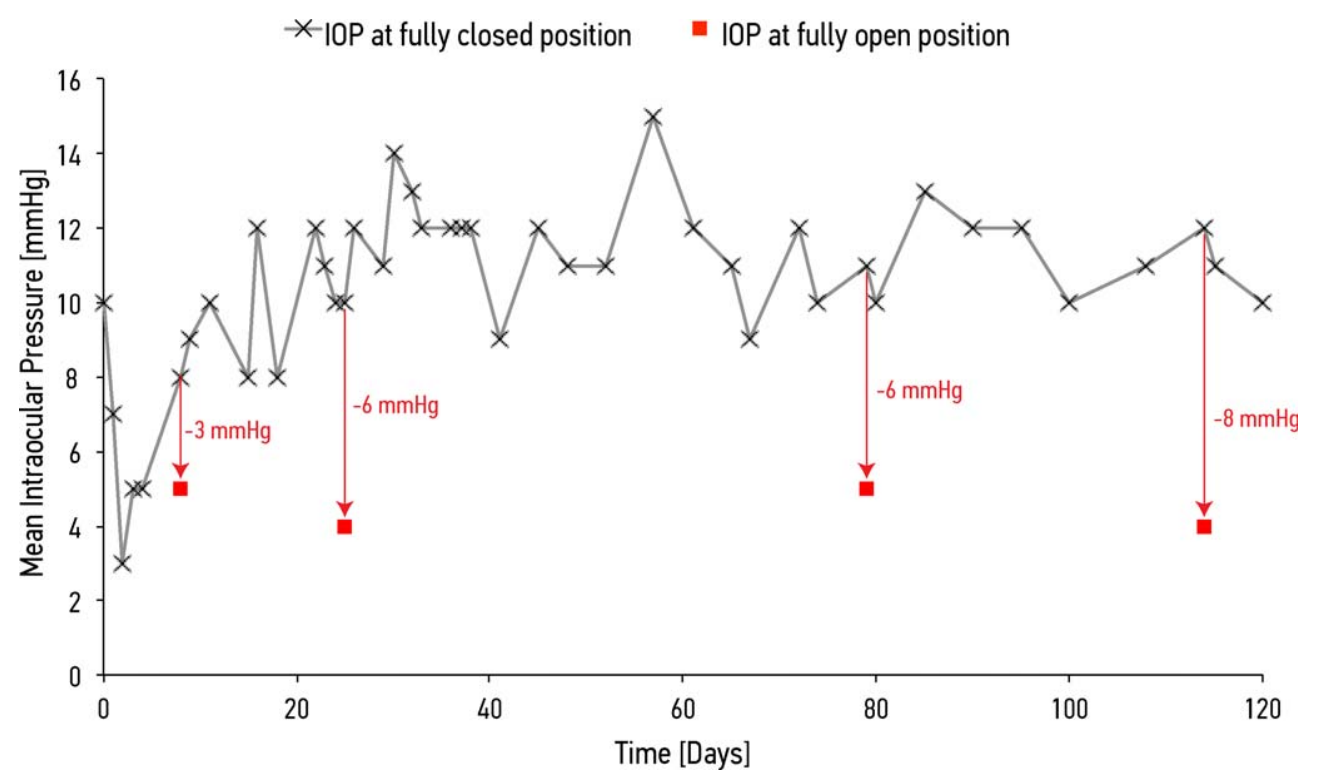

Figure 4. Plot of the mean IOP over time and the AGDD in its functionally closed position. Once a month, the AGDD was fully opened and the resulting pressure was measured (red square symbol). The arrows help visualize the magnitude of the pressure drop when the device was adjusted from its fully closed to its fully opened position.

Results showed that after a week (8 days, Fig. 3) the difference in IOP between control and operated eyes was no longer significant. For the eyes implanted with the AGDD, the IOP went back to preoperative level after only a few days following surgery and the hypotony was minimized $(<4$ days). This short recovery time after the filtering procedure results from the fact that the AGDD was inserted in its functional closed position, thus, limiting outflow to the leakages around the nozzle and allowing the eye to resume the initial IOP level. However, the resistance to outflow regained the preoperative level as a result of the paracentesis better accommodating the diameter of the nozzle to a watertight fit. In humans, this process would certainly have a different time profile.

The use of this AGDD as a modular flow resistance is most efficient in the early stages after implantation. Following the closed position. surgical procedure the AGDD could be set into a functional closed position to minimize hypotony. Once the clinically relevant IOP is reached, the resistance to outflow could be adjusted in the early postoperative phase up to 2 to 3 months after surgery.

The 4-month follow-up may be too short to fully evaluate the mid- to long-term safety and efficacy profile of this AGDD. This is the main limitation of this study. Further clinical evaluations are planned to address these issues. Furthermore, such filtering surgery using glaucoma drainage devices might suffer from postoperative scarring processes. This would likely occur in a late postoperative stage and as such might impede aqueous outflow around the device, despite a patent and functional tube. To address this issue, alternative ducting

$\square$ IOP at open position

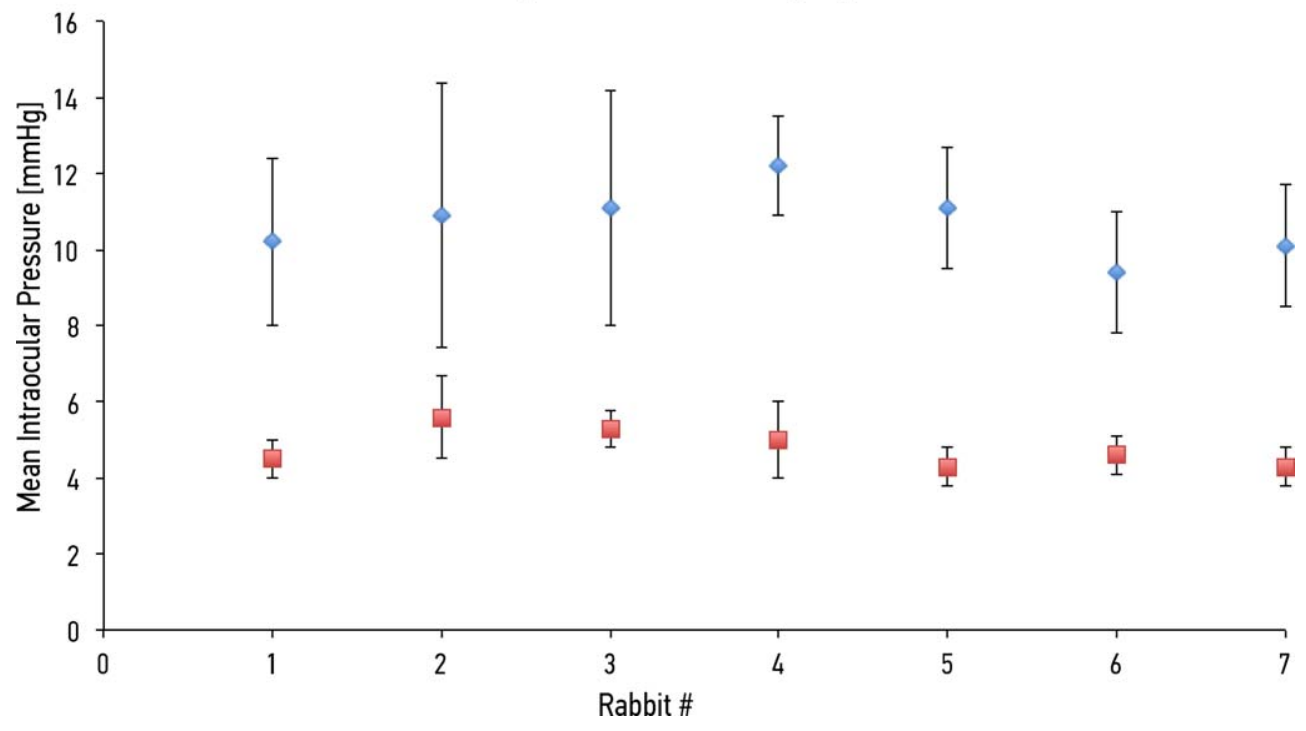

Figure 5. Plot of the mean IOP drop for all rabbits. The pressure drop was significant between the fully opened position of the AGDD and the fully 


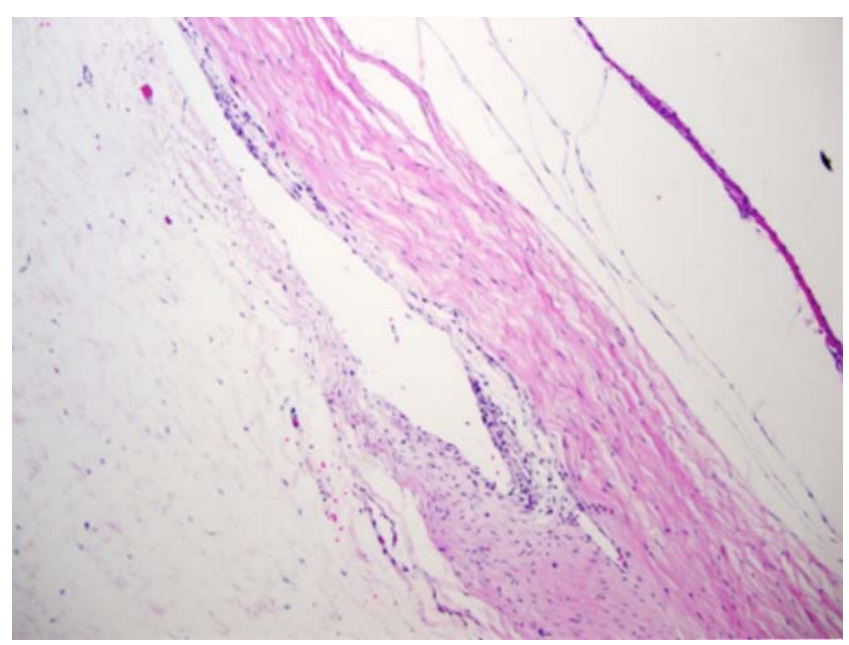

Figure 6. Histopathologic analysis demonstrated a cavity lined by flattened cells surrounded by few lymphocytes. Circumscribed postsurgical cicatricial remodeling can be observed inferior to the cavity in the dense connective tissue of the sclera. Original magnification: $\times 63$, hematoxylin-eosin.

routes to more posterior drainage area, such as the orbital cone, might be envisioned.

Critical aspects, such as safety and efficiency of the AGDD, were assessed in this first in vivo study. The rate of aqueous humor outflow was easily adjustable during the entire postoperative period based on the control of the outflow resistance of the AGDD between the fully closed and fully open positions. The surgical technique to implant the AGDD is comparable to the Ex-PRESS procedure, demonstrating the simplicity and relative ease of use of the implant. These first in vivo results provided encouraging data on the safety and performance of the device. A human clinical trial will follow to demonstrate all aspects of safety, performance, and efficacy of the AGDD in glaucoma patients. This will provide an effective means of controlling IOP during the initial postoperative period on a per patient basis, in the aim to possibly minimize

\section{Acknowledgments}

Disclosure: A. Villamarin, None; N. Stergiopulos, None; S. Bigler, None; A. Mermoud, None; A. Moulin, None; S. Roy, None

\section{References}

1. Congdon N, O'Colmain B, Klaver CC, et al. Causes and prevalence of visual impairment among adults in the United States. Arch Ophthalmol. 2004;122:477-485.
2. Quigley HA, Broman AT. The number of people with glaucoma worldwide in 2010 and 2020. Br J Ophthalmol. 2006;90:262267.

3. Ramulu PY, Corcoran KJ, Corcoran SL, Robin AL. Utilization of various glaucoma surgeries and procedures in Medicare beneficiaries from 1995 to 2004. Ophthalmology. 2007;114: 2265-2270.

4. Joshi AB, Parrish RK 2nd, Feuer WF 2002 survey of the American Glaucoma Society: practice preferences for glaucoma surgery and antifibrotic use. J Glaucoma. 2005;14:172174 .

5. Desai MA, Gedde SJ, Feuer WJ, Shi W, Chen PP, Parrish RK 2nd. Practice preferences for glaucoma surgery: a survey of the American Glaucoma Society in 2008. Ophthalmic Surg Lasers Imaging. 2011;42:202-208.

6. Gedde SJ, Herndon LW, Brandt JD, Budenz DL, Feuer WJ, Schiffman JC. Postoperative complications in the tube versus trabeculectomy (TVT) study during five years of follow-up. $A m$ J Ophthalmol. 2012;153:804-814.

7. Gedde SJ, Schiffman JC, Feuer WJ, Herndon LW, Brandt JD, Budenz DL. Three-year follow-up of the tube versus trabeculectomy study. Am J Ophthalmol. 2009;148:670-684.

8. Kee C. Prevention of early postoperative hypotony by partial ligation of silicone tube in Ahmed glaucoma valve implantation. J Glaucoma. 2001;10:466-469.

9. Schwartz KS, Lee RK, Gedde SJ. Glaucoma drainage implants: a critical comparison of types. Curr Opin Opbthalmol. 2006;17: 181-189.

10. Villamarin A, Roy, S, Bigler, S, Stergiopulos, N. A new adjustable glaucoma drainage device. Invest Ophthalmol Vis Sci. 2014;55:1848-1852

11. Stergiopulos N. Non-invasively adjustable drainage device. US Patent. 2011;12:743-955.

12. Bigler SSN. Apparatus and methods for treating excess intraocular fluid. US Patent. 2012;13:349-353.

13. Dahan E, Carmichael TR. Implantation of a miniature glaucoma device under a scleral flap. J Glaucoma. 2005;14: 98-102.

14. Bouhenni RA, Dunmire J, Sewell A, Edward DP. Animal models of glaucoma. J Biomed Biotechnol. 2012;2012:692609.

15. Werner L, Chew J, Mamalis N. Experimental evaluation of ophthalmic devices and solutions using rabbit models. Vet Ophthalmol. 2006;9:281-291.

16. Samsel PA, Kisiswa L, Erichsen JT, Cross SD, Morgan JE. A novel method for the induction of experimental glaucoma using magnetic microspheres. Invest Ophthalmol Vis Sci. 2011;52:1671-1675.

17. Ruiz-Ederra J, Verkman AS. Mouse model of sustained elevation in intraocular pressure produced by episcleral vein occlusion. Exp Eye Res. 2006;82:879-884.

18. Shareef SR, Garcia-Valenzuela E, Salierno A, Walsh J, Sharma SC. Chronic ocular hypertension following episcleral venous occlusion in rats. Exp Eye Res. 1995;61:379-382.

19. de Jong LA. The Ex-PRESS glaucoma shunt versus trabeculectomy in open-angle glaucoma: a prospective randomized study. Adv Ther. 2009;26:336-345. 